\title{
BEM calculation of the complex thermal impedance of microelectronic devices
}

\author{
B. Vermeersch*, G. De Mey \\ Department of Electronics and Information Systems, Ghent University, Sint Pietersnieuwstraat 41, 9000 Gent, Belgium
}

Received 13 July 2006; accepted 19 September 2006

Available online 22 November 2006

\begin{abstract}
This paper presents a numerical method for modelling the dynamic thermal behaviour of microelectronic structures in the frequency domain. A boundary element method (BEM) based on a Green's function solution is proposed for solving the 3D heat equation in phasor notation. The method is capable of calculating the AC temperature and heat flux distributions and complex thermal impedance for packages composed of an arbitrary number of bar-shaped components. Various types of boundary conditions, including thermal contact resistance and convective cooling, can be taken into account. A simple benchmark case is investigated and a good convergence towards the analytical solution is obtained. Simulation results for a thin plate under convective cooling are compared with a theoretical model and an excellent agreement is observed. In a second example a more complicated three-layer structure is investigated. The BEM is used to analyse the thermal behaviour if delamination of the package occurs, and a physical explanation for the results is given.
\end{abstract}

(C) 2006 Elsevier Ltd. All rights reserved.

Keywords: Thermal impedance; Microelectronics; Boundary element method; Green's function; Nyquist plot; Heat transfer; Phasor notation

\section{Introduction}

For thermal steady-state characterization of electronic packages, the thermal resistance $R_{t h}$ is commonly used. By definition this is nothing else than the junction temperature divided by the power dissipated in the same junction. It is hereby assumed that the ambient air is at a zero reference temperature, in other words the temperature difference between junction and ambient has to be used.

Recently, the dynamic (time-dependent) characterization of electronic packages is receiving more and more attention. Due to the small device dimensions, thermal phenomena are relatively fast, in contrast to most other practical heat transfer applications. The dynamic characterization is particularly interesting with respect to the reliability of the electronic devices [1,2].

In some text books on heat transfer, a part of the chapter "conduction" is devoted to time dependent problems [3,4]. The transient behaviour of a circuit can be described in

\footnotetext{
${ }^{*}$ Corresponding author. Tel.: +3292648952 ; fax: +3292648961.

E-mail addresses: bjorn.vermeersch@elis.ugent.be (B. Vermeersch), demey@elis.ugent.be (G. De Mey).
}

terms of its thermal step or impulse response. This models usually require a lot of data, and various attempts have been made to make them more compact with sufficient accuracy [5,6]. In many electronic devices (e.g. digital circuits) however, electrical signals and hence the power dissipation is periodical. This suggests a thermal characterization in the frequency domain rather than in the time domain. The concept of thermal resistance can be easily extended for dynamic characterization when using phasor notation $(\partial / \partial t \rightarrow \mathrm{j} \omega)$. Assuming from now on that the ambient air is at zero reference temperature, we have

$Z_{t h}(\mathrm{j} \omega)=\frac{T(\mathrm{j} \omega)}{P(\mathrm{j} \omega)}$,

where $Z_{t h}$ is the thermal impedance (in $\mathrm{K} / \mathrm{W}$ ), $T$ and $P$ the temperature and power phasor, respectively, and $\omega=2 \pi f$ the angular frequency of the heat source (in $\mathrm{rad} / \mathrm{s}$ ). While phasor notation (AC analysis) is quite common in electric and electronic circuits, the application in the field of thermal analysis seems rather exceptional. Book sections tackling thermal AC behaviour are unexisting, and only a limited number of papers is devoted to it [7-9]. 
Although the transient behaviour (e.g. the response to a heat power step) can still be obtained if necessary using Fourier techniques, it should be stressed here that we are focussing on a thermal characterization directly in the frequency domain. The main reason is that the complex thermal impedance $Z_{t h}(\mathrm{j} \omega)$ is showing some interesting and powerful properties which are not revealed in the time domain, as will be explained in a moment. In addition, $Z_{t h}$ can be easily measured experimentally for microelectronic devices.

Several BEM techniques for transient thermal analysis have been published in the literature [10-15], however these are using time-stepping schemes and the results are obviously represented in the time domain. In some of the papers $[11,13]$ the governing boundary integral is solved in the Laplace domain. However, the results are transformed back to the time domain with a numerical method for Laplace inversion using real values for the Laplace variable $s$. Only one paper specifically dedicated to electronic packages was found [12]. Again the analysis was carried out in the time domain, for a two-dimensional instead of three-dimensional geometry.

The thermal impedance is a complex function of the frequency. For a graphical representation, a Nyquist plot (i.e $\operatorname{Im}\left[Z_{t h}(\mathrm{j} \omega)\right]$ versus $\operatorname{Re}\left[Z_{t h}(\mathrm{j} \omega)\right]$ with $\omega$ as a parameter) is used. Such an impedance curve provides a complete dynamic description and can hence be seen as a thermal blueprint of the structure. Recently it has been observed both experimentally and theoretically that the Nyquist curve of the thermal impedance is in most cases composed of a small number (2-5) of circular arcs [16-19]. Because of this remarkable geometrical property only a very limited number of parameters is necessary to describe the impedance function with high accuracy [19]. Hence $Z_{t h}(\mathrm{j} \omega)$ provides a model which is both compact and complete, making it an interesting tool for dynamic thermal characterization of electronic devices.

In this contribution, a method for the numerical calculation of the thermal impedance in the frequency domain is proposed. The electronic devices are modelled by a number of bar shaped layers which can be placed next to and on top of each other. Because most microelectronic devices are produced using planar technologies (integrated circuits, thin film resistors, ...) the heat sources are essentially two-dimensional. Hence the heat dissipation can be taken into account by injecting a certain heat flux either at the surface of one material layer or in the interface between two layers. For the calculation of the thermal impedance only the temperature inside the heat source is needed. Since the source is located at a material surface the knowledge of the temperature distribution inside the layers is not necessary. This explains why the boundary element method is highly attractive for this application. Both running time and computer storage will be considerably smaller as compared to a finite element method.

The rest of the paper is organized in the following way. First a boundary integral for the heat conduction equation in phasor notation is obtained (Section 2). In the next section it is shown in detail how this equation can be solved numerically. First a method for a single bar-shaped layer is presented. The method is then extended for multi-layered structures. A good agreement between numerical data and analytic results is observed. Next, simulation results for various package configurations are presented and discussed (Section 4). A summary, given in Section 5, concludes the paper.

\section{Basic theory}

Let us consider a structure composed of a finite number of bar-shaped components. The different parts can be stacked on top of each other as layers but can be in sidealong contact as well. No proportional or structural limitations are imposed: each component can have different dimensions and may represent a different material. The only assumption made is that each barshaped piece is oriented along the $x, y$ and $z$ directions. An example is shown in Fig. 1.

The structures just described will be used as a model for microelectronic devices. In Fig. 1 the layers 1, 2 and 4 could e.g. represent a semiconductor, bonding material and ceramic substrate, respectively. Each component is described by means of two thermal parameters, namely its thermal conductivity $k$ (in $\mathrm{W} / \mathrm{mK}$ ) and heat capacity per volume unit $C_{v}$ (in $\mathrm{J} / \mathrm{m}^{3} \mathrm{~K}$ ). It is assumed that the materials used in the structure are isotropic. In other words, $k$ and $C_{v}$ are scalar values which are constant throughout the entire volume of each bar-shaped layer. Since for non-linear systems strictly speaking a thermal impedance cannot be defined, non-linear effects such as the temperature dependence of the thermal conductivity are not taken into account.

Let us now focus on a single bar-shaped piece of material, which e.g. may be one of the components in a multi-layered structure. In phasor notation, the temperature distribution $T$ (in $\mathrm{K}$ ) is given by

$k \nabla^{2} T(\vec{r})-\mathrm{j} \omega C_{v} T(\vec{r})=-p$,

where $p$ is the power density phasor (in $\mathrm{W} / \mathrm{m}^{3}$ ) and $\mathrm{j}=$ $\sqrt{-1}$ the imaginary unit. As mentioned before, the heat sources in microelectronic applications can be assumed to be located at the surface of one or more materials, hence $p \equiv 0$. Two-dimensional surface heat sources can be easily

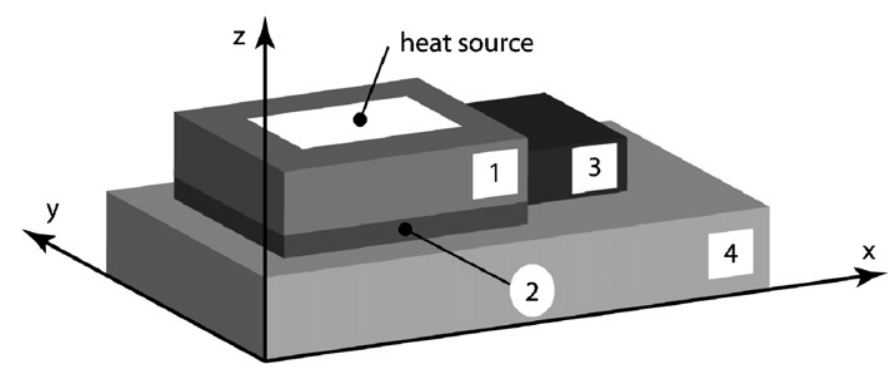

Fig. 1. Structure consisting of four bar-shaped components. 
taken into account by the boundary conditions, as will be shown in Section 3.1. The fundamental solution of Eq. (2) for a Dirac impulse excitation, i.e. the so-called Green's function, is found to be [20]:

$G\left(\vec{r} \mid \vec{r}^{\prime}\right)=\frac{1}{4 \pi k\left|\vec{r}-\vec{r}^{\prime}\right|} \exp \left(-\sqrt{\frac{j \omega C_{v}}{k}}\left|\vec{r}-\vec{r}^{\prime}\right|\right)$,

where $\vec{r}$ and $\vec{r}^{\prime}$ are the field and source point, respectively. Using the divergence theorem (Gauss), the following boundary integral equation is obtained:

$T\left(\vec{r}^{\prime}\right)=k \iint_{\mathscr{S}}\left(G\left(\vec{r} \mid \vec{r}^{\prime}\right) \frac{\partial T(\vec{r})}{\partial n}-T\left(\vec{r}^{\prime}\right) \frac{\partial G\left(\vec{r} \mid \vec{r}^{\prime}\right)}{\partial n}\right) \mathrm{d} S$,

where $\mathscr{S}$ is the surface of the material, i.e. the union of the six sides of the bar. $\partial . / \partial n$ denotes the normal derivative with respect to a unit vector $\vec{n}$ perpendicular to the surface and which is, by convention, pointing out of the material. Due to the bar-shaped geometry and its particular orientation, the normal vector $\vec{n}$ is for each of the six sides directed either along or opposite to one of the coordinate axes. The derivatives of the Green's function with respect to these directions can be calculated analytically and are given by

$$
\begin{aligned}
\frac{\mathrm{d} G\left(\vec{r} \mid \vec{r}^{\prime}\right)}{\mathrm{d} u}= & -\frac{u-u^{\prime}}{4 \pi k\left|\vec{r}-\vec{r}^{\prime}\right|^{3}} \exp \left(-\sqrt{\frac{\mathrm{j} \omega C_{v}}{k}}\left|\vec{r}-\vec{r}^{\prime}\right|\right) \\
& \cdot\left[1+\sqrt{\frac{\mathrm{j} \omega C_{v}}{k}}\left|\vec{r}-\vec{r}^{\prime}\right|\right] .
\end{aligned}
$$

The normal derivative is easily obtained by replacing $u\left({ }^{\prime}\right)$ with $x\left({ }^{\prime}\right), y\left({ }^{\prime}\right)$ or $z\left({ }^{\prime}\right)$ and adding the appropriate sign: $\partial G\left(\vec{r} \mid \vec{r}^{\prime}\right) / \partial n= \pm \mathrm{d} G\left(\vec{r} \mid \vec{r}^{\prime}\right) / \mathrm{d} u$.

\section{Boundary element method}

In this section an algebraic set for Eq. (4) is obtained numerically by discretizing the boundary $\mathscr{S}$ of the structure. From the set the temperature can be derived, which eventually leads to the thermal impedance $Z_{t h}$. For practical reasons and sake of simplicity, a shorter notation for the normal derivative of the temperature will be used:

$\Theta(\vec{r}) \equiv \frac{\partial T(\vec{r})}{\partial n}$.

\subsection{Single layer structures}

First the procedure for a single bar-shaped component is explained. The method can be further extended for structures containing two or more layers, as described later on in Section 3.2.

As mentioned before, the structure is oriented along the $x, y$ and $z$ directions. The according dimensions of the component are noted as size $X$, size $Y$ and size $Z$, respectively. The discretization of the boundary $\mathscr{S}$ is

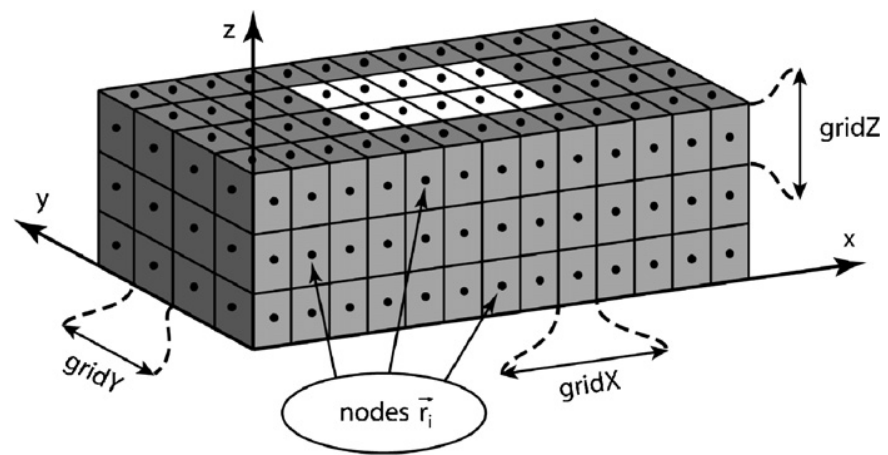

Fig. 2. Division of a bar-shaped component into discrete boundary segments.

presented in Fig. 2. The six sides of the bar are divided by a grid with spacing grid $X$, grid $Y$ and grid $Z$, leading to a number ( $N$ in total) of rectangular segments. It is clear that the dimensions of the component and the heat source(s) must be integer multiples of the grid parameters, and alignment of the source(s) with the grid is necessary.

The centre points of the segments are noted as $\vec{r}_{i}$, with $i$ the segment number satisfying $1 \leqslant i \leqslant N$, and will be referred to as "nodes". It is now assumed that for each segment the temperature $T$ and the normal heat flux $q_{n}=$ $-k \Theta$ is uniform, and equals the value obtained in the node. Under these assumptions the evaluation of Eq. (4) in the $j$ th node (i.e. $\vec{r}=\vec{r}_{j}$ ) can be written as:

$T\left(\vec{r}_{j}\right)=T_{j}=k \sum_{i=1}^{N} \Theta_{i} \cdot \Phi_{i, j}-k \sum_{i=1}^{N} T_{i} \cdot \Psi_{i, j}, \quad j=1 \ldots N$,

where $\Phi$ and $\Psi$ have been introduced as short integral notations, namely

$\Phi_{i, j}=\iint_{\mathscr{S}_{i}} G\left(\vec{r} \mid \vec{r}_{j}^{\prime}\right) \mathrm{d} S$

and

$\Psi_{i, j}=\iint_{\mathscr{S}_{i}} \frac{\partial G\left(\vec{r} \mid \vec{r}_{j}^{\prime}\right)}{\partial n} \mathrm{~d} S$

with $\mathscr{S}_{i}$ the $i$ th segment. The $\Phi$ and $\Psi$ integrals are, due to the complicated form of the Green's function and its normal derivative, not available in closed form but must be calculated numerically. For this purpose each segment is split into a uniform rectangular grid, as shown in Fig. 3.

Both horizontally and vertically $\mathscr{K}$ divisions are applied, leading to $\mathscr{K}^{2}$ subsegments. It is recommended to choose an odd value for $\mathscr{K}$. This ensures that one of the subsegments is centred around the node of the segment which it belongs to. The latter can be important for convergence issues and for taking the contribution of a node to its own segment correctly into account, as will be explained further. The integrals (8) and (9) can now be 


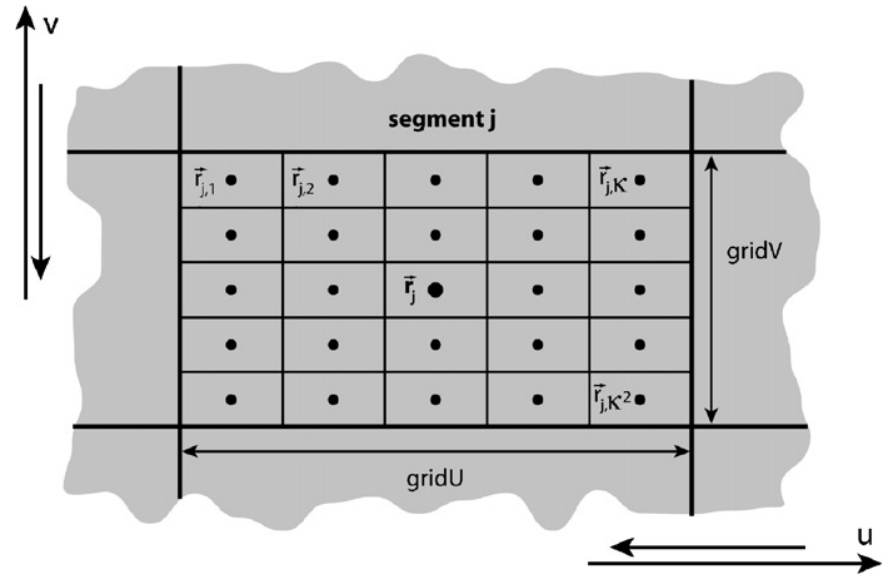

Fig. 3. Division of a segment into $\mathscr{K}^{2}$ subsegments for numerical calculation of the $\Phi$ and $\Psi$ integrals (in this example $\mathscr{K}=5$ ).

approximated as

$\Phi_{i, j} \approx \frac{\operatorname{grid} U \cdot \operatorname{grid} V}{\mathscr{K}^{2}} \sum_{\kappa=1}^{\mathscr{K}^{2}} G\left(\vec{r}_{j, \kappa} \mid \vec{r}_{i}^{\prime}\right)$,

$\left.\Psi_{i, j} \approx \frac{\operatorname{grid} U \cdot \operatorname{grid} V}{\mathscr{K}^{2}} \sum_{\kappa=1}^{\mathscr{K}^{2}} \frac{\partial G\left(\vec{r} \mid \vec{r}^{\prime}\right)}{\partial n}\right|_{\left(\vec{r}_{j, k} \mid \vec{r}_{i}^{\prime}\right)}$

in which $\vec{r}_{j, \kappa}$ denotes the centre point of the $\kappa$ th subsegment in the $j$ th segment. $U$ and $V$ must be substituted by two out of three choices $(X, Y, Z)$ according to the coordinates varying over the $j$ th segment, as illustrated in Fig. 3.

Some precautions should be taken while using Eqs. (10) and (11), namely where $i=j$. For those cases, a singularity occurs in the Green's function (3) as well as in its normal derivative (5) for the subsegment with number $\kappa^{*}=\left(\mathscr{K}^{2}+1\right) / 2$. It was hereby assumed that $\mathscr{K}$ is odd, as recommended. Taking the value $G\left(\vec{r}_{i, \kappa^{*}} \mid \vec{r}_{i}^{\prime}\right)$ (and similar for $\partial G / \partial n$ ) has no mathematical nor physical meaning; a more profound analysis is needed for integration over this particular subsegment. The method consists of separating the Green's function into a singular and a regular part. For the former the integration can be carried out analytically [21]. Further details will be omitted here as the singular term is essentially the same as found for electrostatic potential problems [22]. The result is that the following substitution in (10) must be made:

$\frac{\operatorname{grid} U \cdot \operatorname{grid} V}{\mathscr{K}^{2}} G\left(\vec{r}_{i, \kappa^{*}} \mid \vec{r}_{i}^{\prime}\right) \rightarrow\left(\mathscr{I}_{1}+\mathscr{I}_{2}\right)$

in which

$$
\begin{aligned}
\mathscr{I}_{1}= & -\frac{l}{\pi k} \ln \left[\tan \left(\frac{\pi}{4}-\frac{1}{2} \arctan \frac{b}{l}\right)\right] \\
& -\frac{b}{\pi k} \ln \left[\tan \left(\frac{\pi}{4}-\frac{1}{2} \arctan \frac{l}{b}\right)\right]
\end{aligned}
$$

and

$$
\begin{aligned}
\mathscr{I}_{2} \approx & \frac{\mathrm{j} \omega C_{v}}{12 \pi k^{2}}\left(b l^{2} \sqrt{1+\left(\frac{b}{l}\right)^{2}}+l^{3} \operatorname{arcsinh}\left(\frac{b}{l}\right)\right) \\
& +\frac{\mathrm{j} \omega C_{v}}{12 \pi k^{2}}\left(l b^{2} \sqrt{1+\left(\frac{l}{b}\right)^{2}}+b^{3} \operatorname{arcsinh}\left(\frac{l}{b}\right)\right) \\
& -(1+\mathrm{j}) \sqrt{\frac{\omega C_{v}}{2 k}} \frac{b l}{\pi k},
\end{aligned}
$$

where $l$ and $b$ are half of the length and width of the subsegment:

$l=\frac{\operatorname{grid} U}{2 \mathscr{K}}, \quad b=\frac{\text { grid } V}{2 \mathscr{K}}$

and $\operatorname{arcsinh}(\alpha)=\ln \left(\alpha+\sqrt{1+\alpha^{2}}\right)$. The result for the integration of the normal derivative does not depend on the dimensions or orientation of the (sub)segment:

$\Psi_{i, i}=-\frac{1}{2 k}, \quad 1 \leqslant i \leqslant N$

Let us now return to the base equation (7). This set of equations contains only temperatures and normal derivatives evaluated at the surface of material, in the form of $N$ node values for both $T$ and $\Theta$. Some of these quantities, however, are known a priori or related with each other, by means of the boundary conditions. The values following directly from the boundary specifications will be noted with a "^" . For a segment covered by a heat source for example, we have

$\hat{\Theta}_{i}=\frac{p_{S}}{k}$

with $p_{S}$ the surface power density (in $\mathrm{W} / \mathrm{m}^{2}$ ) of the heat source. The total power phasor, needed in Eq. (1), is then obviously $P=A_{S} \cdot p_{S}$ with $A_{S}$ the total area of the heat source. For thermal impedance calculations, the bottom of the structure is perfectly cooled (perfect heat sink at ambient temperature):

$\hat{T}_{i}=0$.

The remaining part of the surface is commonly assumed to be thermally isolated (adiabatic boundary condition):

$\hat{\Theta}_{i}=0$.

Convective cooling will not be considered at this moment, but can be taken into account as well, as shown in Section 3.2. Any possible combination of the three types of boundary conditions (17), (18) and (19) leads to the conclusion that for each segment either the temperature or its normal derivative is known a priori. Let us now organize the numeration of the segments, which is arbitrary anyhow, in such a way that the segments for which $\Theta$ is known are assigned the lowest segment numbers, $1 \leqslant i \leqslant M$. The remaining $N-M$ segments where $T$ is known receive numbers $M+1 \ldots N$. Eq. (7) 
can now be written as

$$
\begin{aligned}
T_{j}= & k \sum_{i=1}^{M} \hat{\Theta}_{i} \Phi_{i, j}+k \sum_{i=M+1}^{N} \Theta_{i} \Phi_{i, j}-k \sum_{i=1}^{M} T_{i} \Psi_{i, j} \\
& -k \sum_{i=M+1}^{N} \hat{T}_{i} \Psi_{i, j}, \quad j=1 \ldots N .
\end{aligned}
$$

This is nothing else than a linear set of $N$ equations with $N$ unknowns. In matrix form:

$$
\overline{\bar{A}} \cdot \bar{X}=\bar{B} \quad \text { with } \bar{X}=\left[T_{1} T_{2} \ldots T_{M} \Theta_{M+1} \Theta_{M+2} \ldots \Theta_{N}\right]^{\mathrm{T}}
$$

in which the superscript ${ }^{\mathrm{T}}$ denotes transposition. The elements for the $\bar{A}$ and $\bar{B}$ matrix can be easily identified in terms of the $\Phi$ and $\Psi$ integrals and the boundary conditions $\hat{T}$ and $\hat{\Theta}$. The set (21) is solved using standard procedures such as Gaussian elimination, after which the unknown temperatures and heat fluxes are obtained. The thermal impedance is then derived from the temperatures in the heat source, which however are normally not uniform. Typically, the average source temperature is used, as this is more representative for experimental measurements [23]. By repeating the entire procedure for various frequencies over a wide range, a Nyquist plot of the thermal impedance is obtained.

\subsection{Multilayer structures}

Let us now consider a more general package, consisting of $N_{L} \geqslant 2$ rectangular layers. These components must be properly orientated along the $x, y$ and $z$ directions as illustrated in Fig. 1. Each component is divided into a number of boundary elements, as described before. However, the grid parameters should be kept constant throughout the entire structure, and the components must be aligned with respect to the chosen grid as well. Both conditions are needed to make sure that the segments in the material interfaces are well in contact and overlap completely.

The surface temperature distribution can be obtained in the following way. Eq. (7) is obviously still holding, and can be applied for each component separately:

$$
\begin{gathered}
T_{j}^{\left(n_{L}\right)}=k^{\left(n_{L}\right)} \sum_{i=1}^{N^{\left(n_{L}\right)}} \Theta_{i}^{\left(n_{L}\right)} \cdot \Phi_{i, j}^{\left(n_{L}\right)}-k^{\left(n_{L}\right)} \sum_{i=1}^{N^{\left(n_{L}\right)}} T_{i}^{\left(n_{L}\right)} \cdot \Psi_{i, j}^{\left(n_{L}\right)}, \\
j=1 \ldots N^{\left(n_{L}\right)}
\end{gathered}
$$

in which the superscript ${ }^{\left(n_{L}\right)}$ denotes "evaluated in the $n_{L}$ th component", with $1 \leqslant n_{L} \leqslant N_{L}$. It should be noted that the simple rule obtained for single-component structures (for each segment either $T$ or $\Theta$ follows from the boundary conditions) is no longer valid. Namely, for the segments in the material interfaces, both temperature and normal derivative are unknown. Hence the total number of unknowns in Eq. (22) exceeds $N^{\left(n_{L}\right)}$. In addition, the $N_{L}$ systems (22) are not independent but coupled with each other due to the material interfaces. The extra equations which are needed for a solution of the heat equation are provided by the boundary equations in the interfaces, as will be demonstrated shortly. As a convention for segments which are in thermal contact, $T$ and $\Theta$ will, respectively, be taken as the "primary" and "extra" unknown. For regions where a convective cooling is applied, the temperature and heat flux are not independent as well, but are related through the heat transfer coefficient $h$ :

$h T+k \Theta=0$.

For such segments clearly both $T$ and $\Theta$ are unknowns, hence the convection zones can be treated in the same way as the material interfaces.

Compared to the simple situation of Section 3.1, an interface segment gives rise to an extra unknown. A couple of contacting segments is symbolically represented in Fig. 4. Due to the convention for the normal vector $\vec{n}$, the heat fluxes $q_{n}=-k \Theta$ are pointing out of the material. Each such couple of contacting segments introduces two extra unknown $\Theta$ variables into the system, hence two additional equations per couple are needed in order to obtain a solution for the thermal distribution. These equations are provided by the boundary conditions in the interface, one for the temperature and one for the heat flux. The most common situations will now be discussed.

Perfect thermal contact: Obviously, in this case the temperature must be continuous:

$T_{A}-T_{B}=0$

while the principle of conservation of energy leads to

$k_{A} \Theta_{A}+k_{B} \Theta_{B}=0$.

Imperfect thermal contact: Due to material impurities, roughness of the surfaces, etc., material interfaces are in general not thermally perfect. The small gap between the two materials will induce a temperature drop. An accurate description of this phenomenon is an entire study on its own [24] and will not be done here. The simplest way to model an imperfect thermal contact is using a thermal contact resistance $r_{c}$ (in $\mathrm{Wm}^{2} / \mathrm{K}$ ). It relates the temperature drop and the heat flux passing through the interface as a proportional factor:

$T_{A}-T_{B}+k_{A} \cdot r_{c} \cdot \Theta_{A}=0$.

As no energy can be stored in the interface, the $\Theta$ variables are still related by Eq. (25). Interpretation of the thermal

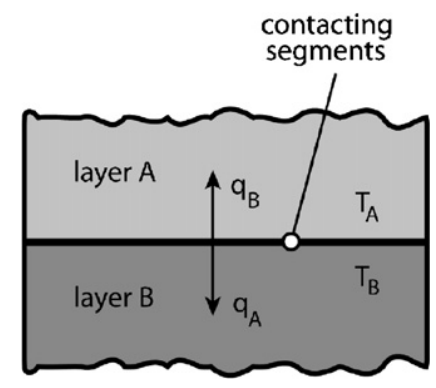

Fig. 4. Cross-section of two contacting segments in a material interface. 
contact resistance is simplified by considering its reciprocal form $r_{c}^{-1}$, which can be easily identified as the thermal conductance per area unit of the interface.

Interface heat source: For a source between two materials, the heat fluxes injected into the layers must in total equal the dissipated power density, hence

$k_{A} \Theta_{A}+k_{B} \Theta_{B}=p_{S}$.

After insertion of the boundary conditions, the algebraic set for the entire structure can be completed and solved using a representation like Eq. (21).

\subsection{Convergence check}

A simple structure, for which the thermal impedance is available in analytic form, will now be investigated. This allows quantization of the convergence behaviour of the BEM simulation by analysing the error for various grid sizes. Concretely, let us consider a substrate, with thickness $H$ and cross-section area $A$, which is completely covered by a uniform heat source. The bottom surface is perfectly cooled, while the side surfaces are thermally isolated. Due to the particular geometry and boundary conditions, the temperature and heat flow are one-dimensional. The thermal impedance can be easily calculated and is found to be

$Z_{t h}=Z_{0} \frac{\tanh \left(\sqrt{\mathrm{j} \omega / \omega_{0}}\right)}{\sqrt{\mathrm{j} \omega / \omega_{0}}}$

where

$Z_{0}=\frac{H}{k S}, \quad \omega_{0}=\frac{k}{C_{v} H^{2}}$.

As an example we will use a silicon substrate $\left(k=160 \mathrm{~W} / \mathrm{mK}, C_{v}=1.784 \times 10^{6} \mathrm{~J} / \mathrm{m}^{3} \mathrm{~K}\right)$ with a cubic shape $($ size $X=$ size $Y=$ size $Z=100 \mu \mathrm{m})$. It should be noted that this particular choice does not limit the applicability of further conclusions. According to (28) the shape of the Nyquist plot remains unaltered for different substrate dimensions or another material, only the impedance and frequency scales have to be adjusted (see Eq. (29)). The result for BEM simulation with a discretization $\quad$ grid $X=$ grid $Y=$ grid $Z=10 \mu \mathrm{m}(N=$ $600)$ and $\mathscr{K}=25$ is shown in Fig. 5.

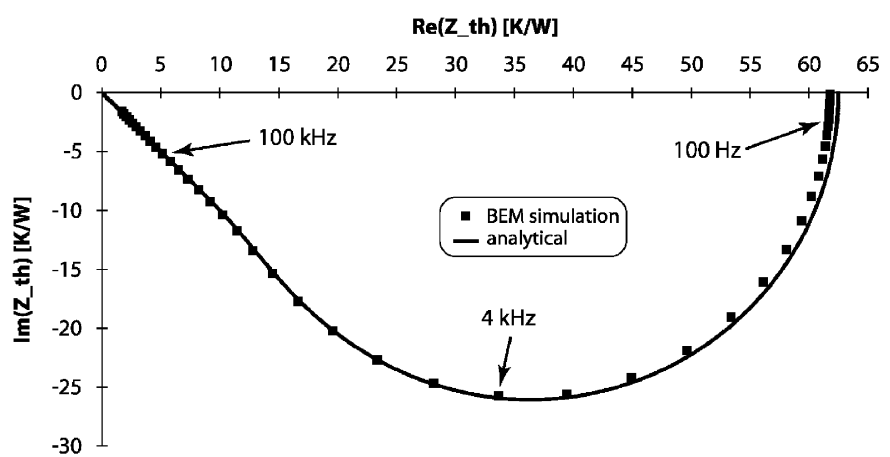

Fig. 5. Calculated thermal impedance for silicon cube.
A good agreement with the analytical solution (28) is observed. The largest discrepancy in absolute terms occurs for low frequencies, however, the relative error for the thermal resistance $R_{t h}=Z_{t h}(\omega=0)$ only amounts to $0.9 \%$. In Fig. 5 three points are marked, at relatively "low" $(f=100 \mathrm{~Hz})$, "middle" $(f=4 \mathrm{kHz})$ and "high" $(f=$ $100 \mathrm{kHz}$ ) frequencies. The impedance for these frequencies is calculated for various square discretizations (grid $X=$ grid $Y=$ grid $Z$ ) with $\mathscr{K}=5$. The relative error for both real and imaginary parts, i.e. $\mid \operatorname{Re}\left(Z_{\text {sim }}\right)-\operatorname{Re}\left(Z_{\text {anal }}\right) /$ $\operatorname{Re}\left(Z_{\text {anal }}\right) \mid$ and $\left|\operatorname{Im}\left(Z_{\text {sim }}\right)-\operatorname{Im}\left(Z_{\text {anal }}\right) / \operatorname{Im}\left(Z_{\text {anal }}\right)\right|$, respectively, is presented in Fig. 6.

The simulation results are clearly converging towards the analytical values as the grid parameters decrease. As indicated in Fig. 6 the error curves can be fitted quite well to a straight line, i.e. a relation of the form $\log ($ error $)=\alpha \cdot \log ($ grid $)+\beta$, where it is noted that $\alpha>1$. In other words the following tendency is observed:

rel. error $\propto g r i d^{\alpha} \Longrightarrow$ rel. error $\propto \frac{1}{\sqrt{N^{\alpha}}}$.

With grid $=5 \mu \mathrm{m}$ an error less than $2 \%$ is obtained, even for the high frequencies.

\section{Numerical experiments}

\subsection{Example 1: thin plate with convective cooling}

Let us consider a plate with length $a$ (z-direction), width $b$ (x-direction) and thickness $t_{s} \ll a, b$ (y-direction) . An example is given in Fig. 7.

One of the sides of the plate is completely covered by a uniform heat source dissipating a power $P$. The plate is subject to a convective cooling, characterized by the heat transfer coefficient $h$ to the ambient air. It has been proven earlier that if the thickness $t_{s}$ is sufficiently small, i.e. $h \cdot t_{s} / k \ll 1$, the temperature is almost uniform in the $y$ direction [3, pp. 52-69]. For such thin plates, the convective heat transfer at the small sides can be neglected compared

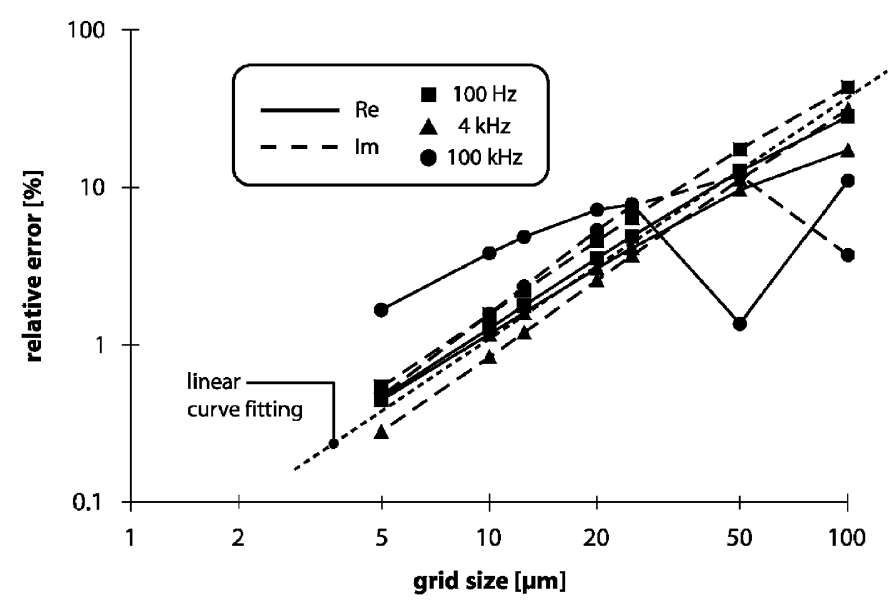

Fig. 6. Relative error for $\operatorname{Re}\left(Z_{t h}\right)$ and $\operatorname{Im}\left(Z_{t h}\right)$ for three frequencies as function of the discretization grid size (double logarithmic scale). 
to the heat removed from the front and rear sides, and adiabatic boundary condition can be used instead. Using these approximations, the one-dimensional temperature distribution satisfies

$\frac{\mathrm{d}^{2} T}{\mathrm{~d} z^{2}}-\frac{1}{L^{2}}\left(1+\frac{\mathrm{j} \omega}{\omega_{0}}\right) T=0$,

in which

$L=\sqrt{\frac{k t_{s}}{2 h}}$ and $\omega_{0}=\frac{2 h}{C_{v} t_{s}}$

are a characteristic length and angular frequency, respectively. The heat source implies

$\left.k b t_{s} \frac{\mathrm{d} T}{\mathrm{~d} z}\right|_{z=a}=P$

while neglecting the convective heat transfer at the other side leads to $\mathrm{d} T /\left.\mathrm{d} z\right|_{z=0}=0$. With these two boundary conditions Eq. (31) can be solved. Finally, the thermal impedance of the plate is then derived from the source temperature $T(z=a)$ :

$Z_{t h}=\frac{L}{k b t_{s}} \frac{\operatorname{cotanh}\left(\sqrt{1+\mathrm{j} \omega / \omega_{0}} \cdot a / L\right)}{\sqrt{1+\mathrm{j} \omega / \omega_{0}}}$.
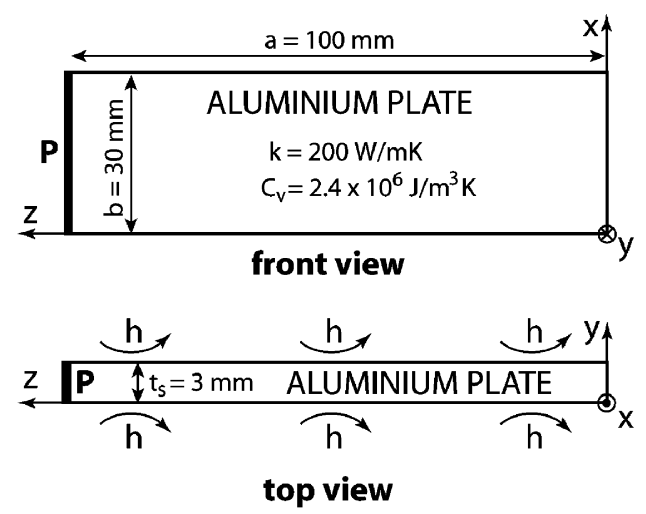

Fig. 7. Heat source on thin aluminium plate with double-sided convective cooling.
This analytical solution can now be used to check numerical results obtained by BEM simulation. As an example the aluminium structure shown in Fig. 7 is analysed for various cooling conditions $(h=10,20$ and $50 \mathrm{~W} / \mathrm{m}^{2} \mathrm{~K}$ ). The discretization of the plate for the numerical calculations was chosen as follows: grid $X=6 \mathrm{~mm}, \quad$ grid $Y=3 \mathrm{~mm}, \quad$ grid $Z=2 \mathrm{~mm} \quad$ and $\mathscr{K}=25$. This leads to 600 segments and 510 additional unknowns are needed for the convection zones. The simulation results are presented in Fig. 8.

One can see clearly that the numerical data correspond almost perfectly with the theoretical model. In addition, the high frequency part of the three impedance curves coincide, thus it is not influenced by the heat transfer coefficient $h$. This leads to the conclusion that convective cooling is only important for relatively slow phenomena.

\subsection{Example 2: three-layer package with delamination}

For the second numerical study, we investigate a typical three-layer electronic package. A chip (semiconductor die) is attached to a ceramic substrate by means of an adhesive (bonding layer). An example of this configuration is shown in Fig. 9. Due to bad chip assembly, aging processes, thermomechanical stress etc. the adhesive layer can start to detach from the substrate [25]. This process, often denoted as "delamination", affects the heat transfer capabilities of the structure and can increase its thermal resistance significantly. The delamination will be modelled by introducing a thermal contact resistance in the adhesive/ substrate interface (ASI).

Let us now assume that a thin air layer is formed between the two materials in the delaminated zone, and that heat can traverse this layer only by conduction. The thermal contact resistance is then easily identified in terms of the thickness and thermal conductivity of the air film:

$r_{c}=\frac{d_{\text {air }}}{k_{\text {air }}}$.

Taking a $500 \mathrm{~nm}$ thick layer and using $k_{\text {air }}=0.025$ leads to $r_{c}=2 \times 10^{-5} \mathrm{Km}^{2} / \mathrm{W}$, which is used for the BEM

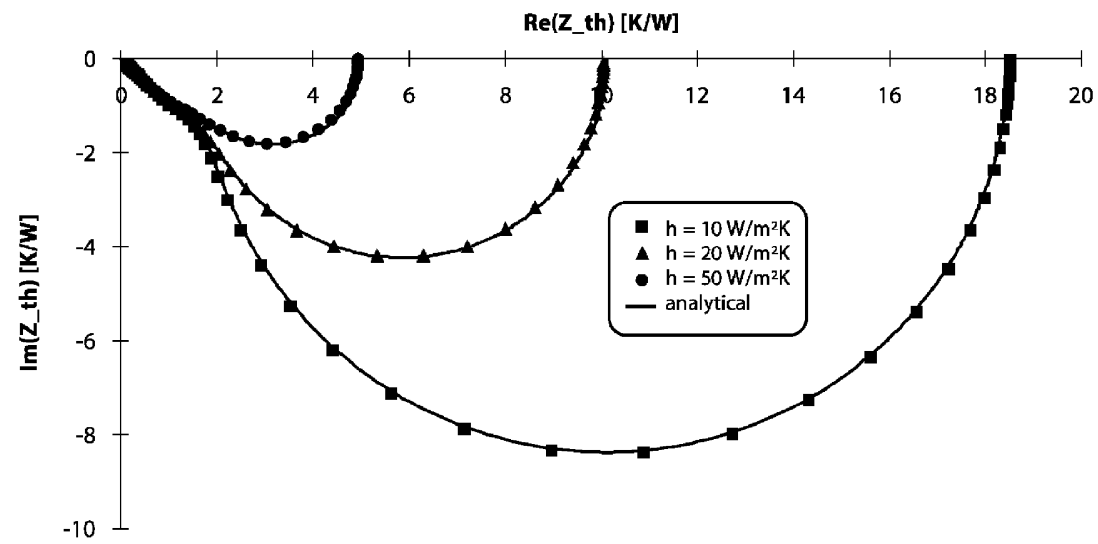

Fig. 8. Calculated thermal impedance for various heat transfer coefficients. 

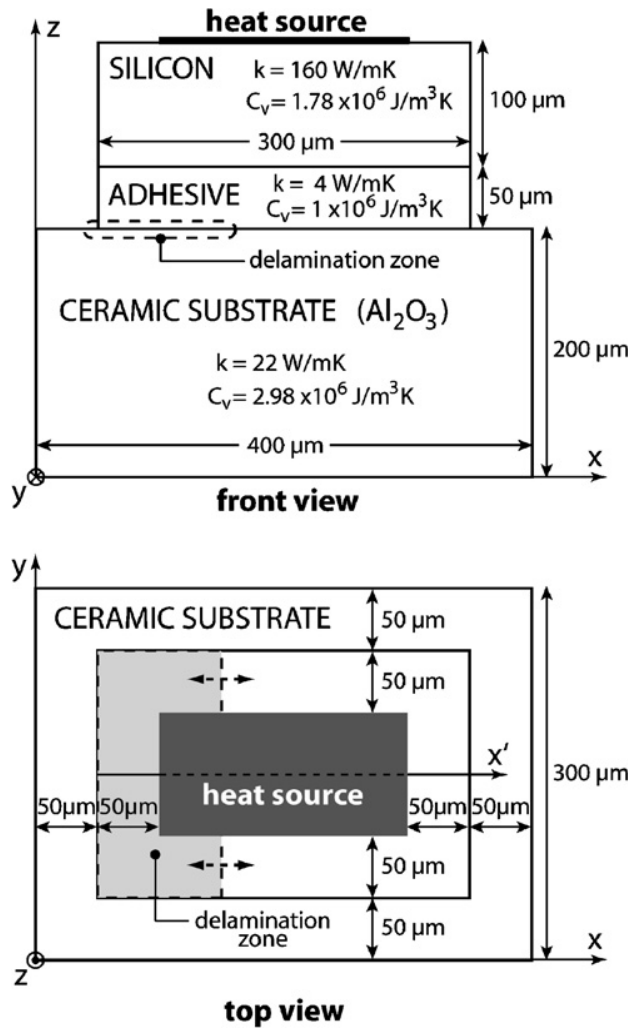

Fig. 9. Package used for delamination simulation.

simulation of the package. Apart from the "normal" package $\left(r_{c}=0\right.$, perfect thermal contact) several delamination stages are considered. The thermal contact resistance is applied over the entire width ( $y$-direction) of the ASI, but for various lengths namely 50,150 and $250 \mu \mathrm{m}$ (see Fig. 9). This corresponds with $13 \%, 50 \%$ and $83 \%$ delamination, respectively. For the simulations, we used grid $X=$ grid $Y=50 \mu \mathrm{m}$, grid $Z=50 \mu \mathrm{m}$ for silicon and $\mathrm{Al}_{2} \mathrm{O}_{3}$, grid $Z=25 \mu \mathrm{m}$ for the adhesive, and $\mathscr{K}=15$. Different grid $Z$ parameters are possible here because there are no material interfaces at the sides of the layers, only at the top and bottom. The resulting thermal impedance curves are presented in Fig. 10.

The shape of the Nyquist curves is almost perfectly circular. A closer look on the high frequency behaviour reveals a second, much smaller circular arc. The impact of the delamination is clearly visible: for the highly delaminated package the thermal resistance increased from 315 to $528 \mathrm{~K} / \mathrm{W}(+68 \%)$. The delamination is visible throughout the entire low frequency arc in the thermal impedance plot. The high frequency part, however, is not influenced by the thermal contact resistance: the small circular arc is the same for each case. This can be explained by taking the decay of the $\mathrm{AC}$ temperature distribution into account. From Eq. (3) it can be seen that the magnitude of the Green's function is approximately decaying in space with a characteristic length $l_{0}=\sqrt{2 k / \omega C_{v}} \propto \omega^{-1 / 2}$. One can therefore expect that for sufficiently high frequencies only

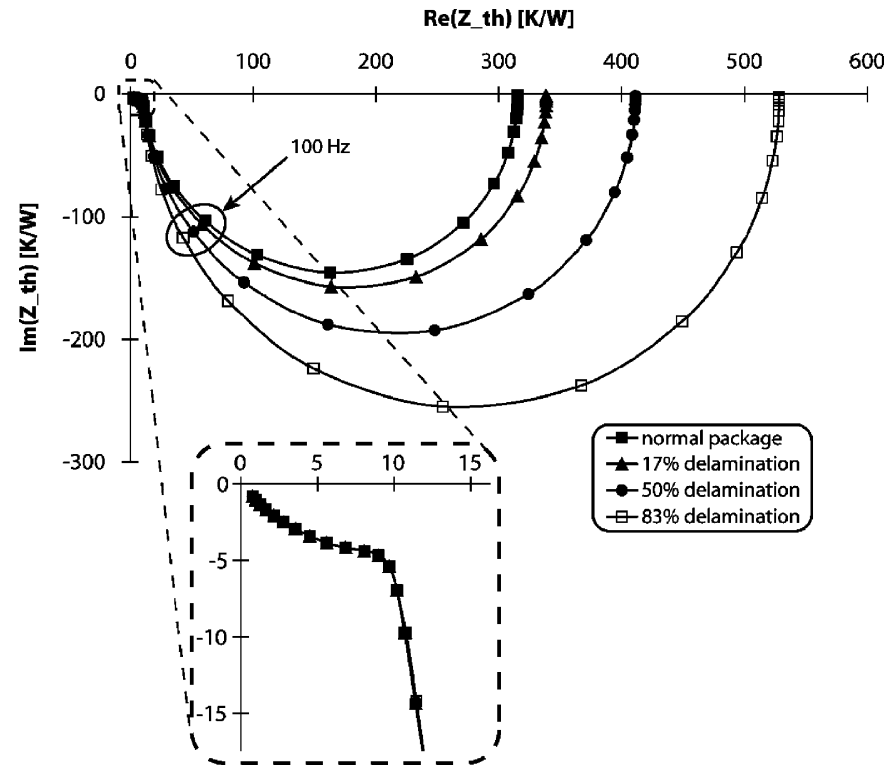

Fig. 10. Calculated thermal impedance of three-layered package for various delamination stages. The inset shows a closer look on the high frequency part.

the upper part of the silicon (regions closest to the source) is considerably heated. The rest of the structure is then almost at ambient temperature. Hence the parameters of these parts, among which the thermal contact resistance of the ASI, hardly play a role in the thermal behaviour.

It is also possible to monitor the heat transfer through the delaminating interface. The magnitudes of the heat flux and temperature are studied along the centre line $x^{\prime}$ (see Fig. 9). The simulation is carried out for steady-state conditions $(f=0, \mathrm{DC})$ and $f=100 \mathrm{~Hz}$ (points marked in Fig. 10). Now grid $X=12.5 \mu \mathrm{m}$ is used in order to increase the resolution along the interface centre line. To facilitate interpretation, the heat flux and temperature curves are normalized, respectively, to the power density $p$ of the source and the DC source temperature $T_{\text {source }}=P \cdot R_{\text {th }}$ where $P=0.2 \mathrm{~W}\left(p=10^{7} \mathrm{~W} / \mathrm{m}^{2}\right)$ was used. The results for the four different delamination stages are shown in Figs. 11-14.

As expected the heat flux is almost uniform for the "normal" package (Fig. 11). Only the values at both sides of the interface deviate from this general tendency. This is a common observation for the spatial derivative of a potential-like quantity (such as $T$ ) in BEM segments close to the edges of the structure [26]. The temperature on the other hand is slightly higher in the middle of the interface because the heat source is centred above the interface and has a smaller area (Fig. 9). When a thermal contact resistance is applied, the delaminated zone can easily be recognized: in this part of the interface the heat flux is smaller and the temperature higher (Figs. 12-14). Particularly noticeable is the extreme value for the heat flux in the segment closest to the delaminated zone. A possible explanation could be that the heat flux lines are bending 


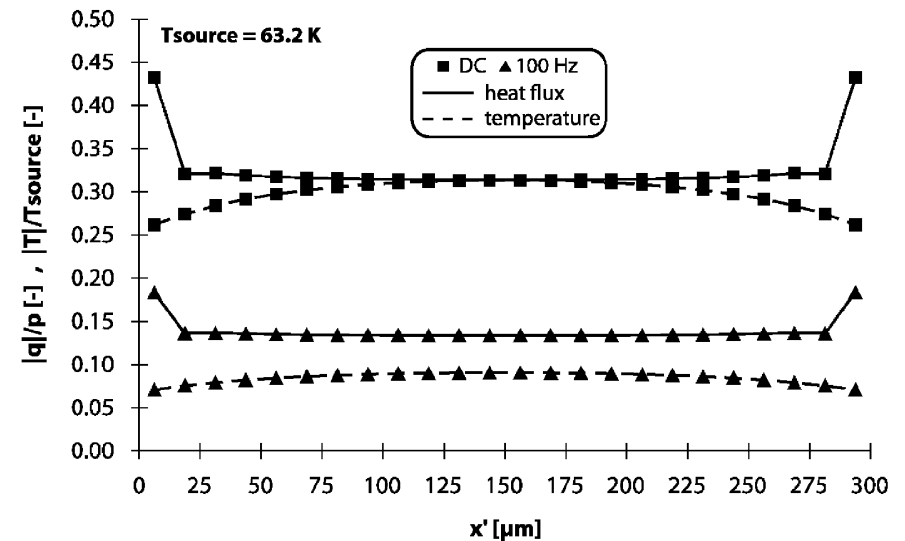

Fig. 11. Normalized heat flux and temperature profiles in ASI for normal package.

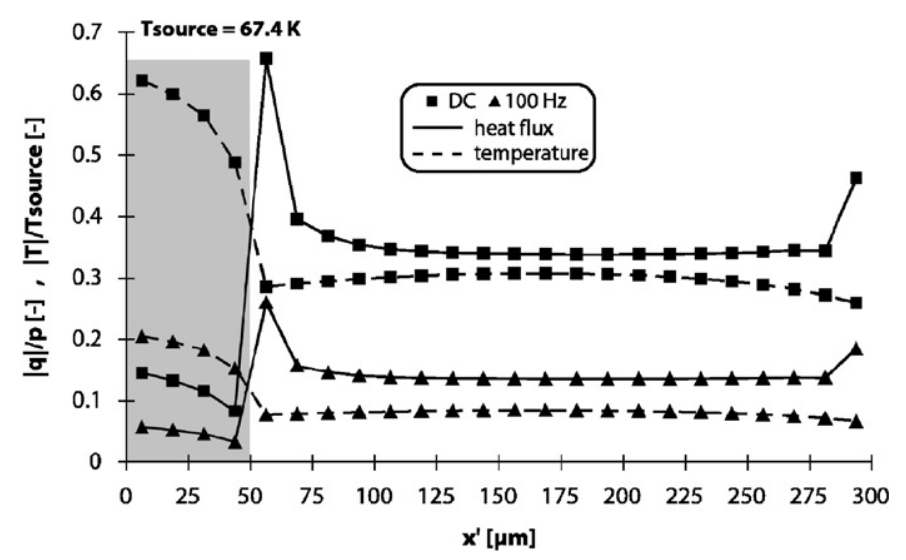

Fig. 12. Normalized heat flux and temperature profiles in ASI for package with $17 \%$ delamination (shaded zone).

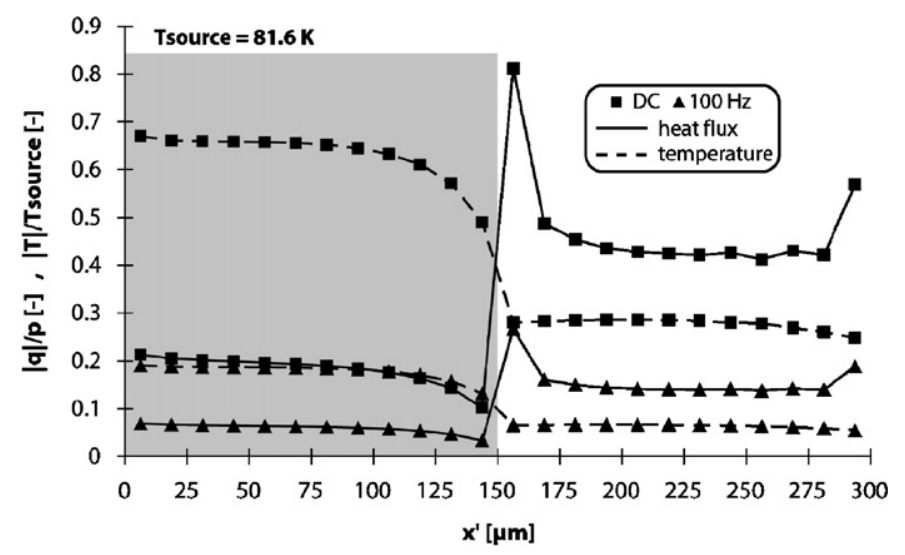

Fig. 13. Normalized heat flux and temperature profiles in ASI for package with $50 \%$ delamination (shaded zone).

away from the interface gap: the badly conducting air film acts as a heat transfer obstacle. This leads to a higher concentration in the immediate neighbourhood of the delamination zone as depicted in Fig. 15.

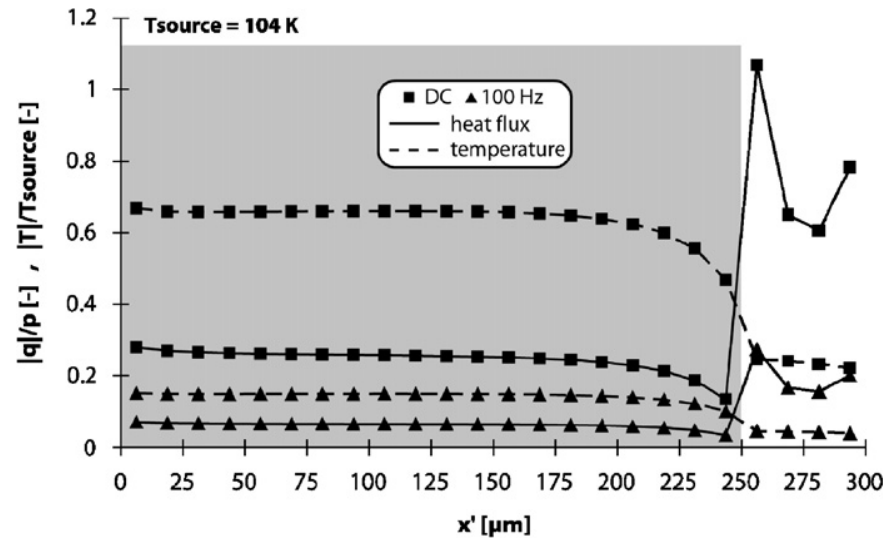

Fig. 14. Normalized heat flux and temperature profiles in ASI for package with $83 \%$ delamination (shaded zone).

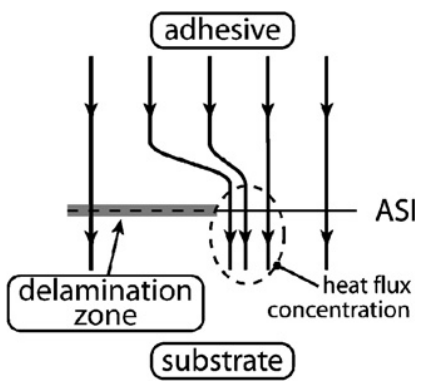

Fig. 15. Bending of heat flux lines leads to higher concentration next to the delamination zone.

\section{Conclusions}

A numerical method for dynamic thermal characterization of electronic packages has been proposed. The importance of such dynamic thermal analysis, and the representation in the frequency domain in particular, was underlined. The complex thermal impedance $Z_{t h}(\mathrm{j} \omega)$, and more precise its Nyquist plot, was shown to be a useful and powerful tool in this respect.

Based on the Green's function, a boundary integral for the heat equation (in phasor notation) was obtained. Next, a boundary element method was presented to solve this equation numerically in a bar-shaped component. The method could easily be extended to multi-layer structures which allows modelling of more complicated package configurations. Various types of boundary conditions were discussed. The accuracy of the method was investigated, and a very good agreement with analytical results was found.

Finally, two practical examples were presented. In the first case study the dynamic thermal behaviour of a thin plate was investigated for various convective cooling situations. Again a nearly perfect match between the numerical BEM results and a theoretical model was observed. In the second example the thermal contact resistance was used to model the delamination process of a three-layered package. A physical explanation for the 
calculation results was given, both for the thermal impedance and temperature and heat flux distribution in the delaminating interface.

\section{Acknowledgements}

B. Vermeersch is preparing a Ph.D. as a Research Assistant for the Research Foundation-Flanders (FWO-Vlaanderen) and likes to thank FWO for supporting the presented work.

\section{References}

[1] Rencz M, Székely V. Determining partial thermal resistances in a heat-flow path with the help of transient measurements. Proceedings of the seventh THERMINIC Workshop (Paris) 24-27 September 2001. p. 250-55.

[2] Székely V. Enhancing reliability with thermal transient testing. Microelectron Reliab 2002;42:629-40.

[3] Bejan A. Heat transfer. New York: Wiley; 1993.

[4] Incopera F, DeWitt D. Introduction to heat transfer. New York: Wiley; 1985.

[5] Székely V. On the representation of infinite-length distributed RC one-ports. IEEE Trans Circuits Syst 1991;38(7):711-9.

[6] Székely V. Identification of RC networks by deconvolution: chances and limits. IEEE Trans Circuits Syst-I Theory Appl 1998;45(3): 244-58.

[7] De Mey G. Integral equation approach to AC diffusion. Int J Heat Mass Transfer 1976;19:702-4.

[8] De Mey G. Thermal conduction in phasor notation. Proceedings of BETECH'95; 1995. p. 1-9.

[9] Vermeersch B, De Mey G. Sinusoidal regime analysis of heat transfer in microelectronic systems. Proceedings of Heat Transfer IX; 2006. p. 449-55.

[10] Wrobel LC, Brebbia CA. A formulation of the boundary element method for axisymmetric transient heat-conduction. Int J Heat Mass Transfer 1981;24(5):843-50.

[11] Banerjee PK. The boundary element methods in engineering. New York: McGraw-Hill; 1994. p. 208-32.
[12] Guven I, Chan CL, Madenci E. Transient two-dimensional thermal analysis of electronic packages by the boundary element method. IEEE Trans Adv Pack 1999;22(3):476-86.

[13] Sutradhar A, Paulino GH, Gray LJ. Transient heat conduction in homogenuous and non-homogenuous materials by the Laplace transform Galerkin boundary element method. Eng Anal Bound El 2002;26:119-32.

[14] Yang MT, Park KH, Banerjee PK. 2D and 3D transient heat conduction analysis by BEM via particular integrals. Comput Methods Appl Mech Eng 2002;191(15-16):1701-22.

[15] Bialecki RA, Jurgas P, Kuhn G. Dual reciprocity BEM without matrix inversion for transient heat conduction. Eng Anal Bound El 2002;26:227-36.

[16] De Mey G, Vermeersch B, Kawka P. Thermal impedance simulations of electronic packages. Proceedings of the 12th international MIXDES conference (Krakow) 22-25 June 2005. p. 267-9.

[17] Vermeersch B, De Mey G. Thermal impedance plots of micro-scaled devices. Microelectron Reliab 2006;46(1):174-7.

[18] Kawka P. Thermal impedance measurements and dynamic modelling of electronic packages. Ph.D. Thesis, Gent, Ghent University; 2005.

[19] Kawka P, De Mey G, Vermeersch B. Thermal characterization of electronic packages using the Nyquist plot of the thermal impedance. IEEE Trans Comp Pack Technol., accepted for publication.

[20] De Mey G. Various applications of the boundary element method. Oradea: University of Oradea, Romania; 2002.

[21] Dean DJ. Thermal design of electronic boards and packages. Ayr: Electrochemical Publications; 1985.

[22] Sladek V, Sladek J, Tanaka M. Evaluation of $1 / \mathrm{R}$ integrals in BEM formulations for 3D problems using coordinate multitransformations. Eng Anal Bound El 1997;20:229-44.

[23] Vermeersch B, De Mey G. Influence of substrate thickness on thermal impedance of microelectronic structures. Microelectron Reliab 2006, doi:10.1016/j.microrel.2006.05.017.

[24] Yovanovich MM. Four decades of research on thermal contact, gap and joint resistance in microelectronics. IEEE Trans Comp Pack Technol 2005;28:182-206.

[25] Sergent J, Krum A. Thermal management handbook. New York: McCraw-Hill; 1998.

[26] Ong ET, Lim KM. Three-dimensional singular boundary elements for corner and edge singularities in potential problems. Eng Anal Bound El 2005;29:175-89. 